\title{
Myotube differentiation in clustered regularly interspaced short palindromic repeat/Cas9-mediated MyoD knockout quail myoblast cells
}

\author{
Si Won Kim, ${ }^{1, \text {, Jeong Hyo Lee }}{ }^{1, a}$, Byung-Chul Park ${ }^{1, *}$, and Tae Sub Park ${ }^{1, *}$
}

\footnotetext{
* Corresponding Authors: Byung-Chul Park Tel: +82-33-339-5792; Fax: +82-33-339-5763, E-mail: bcpark@snu.ac.kr

Tae Sub Park

Tel: +82-33-339-5721; Fax: +82-33-339-5763;

E-mail: taesubpark@snu.ac.kr
}

Graduate School of International Agricultural Technology and Institute of Green-Bio Science and Technology, Seoul National University, Pyeongchang 25354, Korea

a These authors contributed equally to this work. Submitted Sept 29, 2016; Revised Oct 8, 2016; Accepted Oct 24, 2016
Objective: In the livestock industry, the regulatory mechanisms of muscle proliferation and differentiation can be applied to improve traits such as growth and meat production. We investigated the regulatory pathway of $\mathrm{MyoD}$ and its role in muscle differentiation in quail myoblast cells.

Methods: The $M y o D$ gene was mutated by the clustered regularly interspaced short palindromic repeat (CRISPR)/Cas9 technology and single cell-derived MyoD mutant sublines were identified to investigate the global regulatory mechanism responsible for muscle differentiation.

Results: The mutation efficiency was $73.3 \%$ in the mixed population, and from this population we were able to establish two QM7 MyoD knockout subline (MyoD KO QM7\#4) through single cell pick-up and expansion. In the undifferentiated condition, paired box 7 expression in $\mathrm{MyoD}$ KO QM7\#4 cells was not significantly different from regular QM7 (rQM7) cells. During differentiation, however, myotube formation was dramatically repressed in MyoD KO QM7\#4 cells. Moreover, myogenic differentiation-specific transcripts and proteins were not expressed in $\mathrm{MyoD}$ KO QM7\#4 cells even after an extended differentiation period. These results indicate that MyoD is critical for muscle differentiation. Furthermore, we analyzed the global regulatory interactions by RNA sequencing during muscle differentiation.

Conclusion: With CRISPR/Cas9-mediated genomic editing, single cell-derived sublines with a specific knockout gene can be adapted to various aspects of basic research as well as in functional genomics studies.

Keywords: Myoblast; CRISPR-Cas9; Knockout; Muscle Differentiation; MyoD

\section{INTRODUCTION}

Improving the growth of animals in the livestock industry, and in poultry farming in particular, has been an important area of research for a long time. Selective-breeding approaches have been used in the poultry industry, resulting in the generation of specialized commercial populations [1,2]. In addition, for a better understanding of skeletal muscle growth, functional genomics has been used to investigate the mechanisms of muscle proliferation and differentiation [3,4].

To investigate the activity and regulatory mechanisms of specific genes and proteins, two main technical approaches, gene transfer and gene knockout, have been utilized [5-7]. Foreign transgenes can be stably introduced into the host genome of avian species $[8,9]$ and more recently, the piggyBac transposon element has been successfully used for highly efficient gene delivery in chickens [6,10]. Specific genes can also be deleted with knockout techniques $[7,11,12]$. The clustered regularly interspaced short palindromic repeat (CRISPR)/Cas9-mediated genomeediting technical platform, a powerful and versatile technology, was recently developed and has been widely applied to functional genomics studies because the conventional knockout strategy 
by homologous recombination is laborious and difficult, with low efficiency [13,14].

$M y o D$ was the first myogenic regulatory gene to be identified [15]. MyoD, which belongs to a family of proteins known as myogenic regulatory factors (MRFs), is expressed in myoblasts and skeletal muscle tissue but not in smooth or cardiac muscle or nonmuscle tissue $[15,16]$. MRFs are basic helix-loop-helix (bHLH) transcription factors that act sequentially in myogenic differentiation $[15,16]$. MyoD is one of the earliest myogenic regulatory factors for the determination and terminal differentiation of skeletal muscle $[17,18]$. MyoD is expressed at low levels in quiescent muscle satellite cells and its expression increases in response to exercise or damage to muscle tissue $[17,18]$. Although MyoD converts undifferentiated myoblasts into myotubes, muscle development is not dramatically altered in MyoD mutant mice, suggesting that functional redundancy of other myogenic factors such as Myf5 and Mrf4 may compensate for dysfunctional activity of MyoD [19,20].

In this study, we knocked out the $M y o D$ gene in quail myoblast cells with the CRISPR/Cas9 system and examined the global regulatory pathway of MyoD during myotube differentiation.

\section{MATERIALS AND METHODS}

\section{Quail myoblast (QM7) cell culture and induction of myotube differentiation}

QM7 cells (American Type Culture Collection, Manassas, VA, USA) were maintained at $37^{\circ} \mathrm{C}$ in an atmosphere of $5 \% \mathrm{CO}_{2}$ and $60 \%$ to $70 \%$ relative humidity with Medium 199 containing $10 \%$ fetal bovine serum (FBS; Invitrogen, Carlsbad, CA, USA), 2\% chicken serum (Sigma-Aldrich, St. Louis, MO, USA), and $1 \times$ antibiotic-antimycotic (Invitrogen, USA) by subculturing the cells at $70 \%$ confluency. To induce myotube differentiation at $90 \%$ confluency, the differentiation medium containing $0.5 \%$ FBS and $1 \times$ antibiotic-antimycotic was changed and half of the medium was replaced with fresh differentiation medium daily.

\section{CRISPR/Cas9-mediated MyoD knockout and}

fluorescence-activated cell sorting

For knockout of the MyoD gene, $7.5 \mu \mathrm{L}$ Lipofectamine $3000 \mathrm{Re}-$ agent was diluted in $250 \mu \mathrm{L}$ OPTI-MEM (Invitrogen, USA), and $2.5 \mu \mathrm{g}$ each of the Cas9- green fluorescent protein (GFP) coexpression plasmid (Sigma-Aldrich, USA) and MyoD guide RNA (gRNA) was mixed with Lipofectamine P3000 Reagent in 250 $\mu \mathrm{L}$ OPTI-MEM at room temperature. After incubation for $5 \mathrm{~min}$, the two mixtures were combined and incubated for an additional $20 \mathrm{~min}$. The complex mixture was gently pipetted and dropped into a six-well plate containing QM7 cells at 70\% to 80\% confluency. After incubation at $37^{\circ} \mathrm{C}$ in $5 \% \mathrm{CO}_{2}$ for $4 \mathrm{~h}$, cells were gently washed with phosphate-buffered saline (PBS) three times, and fresh culture medium was added. One day after lipofection, GFP-expressing cells were sorted using a FACSAria III cell sorter (Becton, Dickinson and Company, Franklin Lakes, NJ, USA). Following harvest using $0.05 \%$ trypsin-ethylenediaminetetraacetic acid (Invitrogen, USA), cells were resuspended in PBS containing $0.1 \%$ bovine serum albumin (BSA) and strained through a 40 $\mu \mathrm{m}$ cell strainer for fluorescence-activated cell sorting (FACS) (Becton, Dickinson and Company, USA). After sorting, the cells were regrown in culture media for subsequent experiments. To isolate single cell-derived sublines, each well-isolated single colony was isolated and subcultured using smooth silicone grease and a cloning cylinder.

\section{Genotyping by T-vector cloning and sequencing}

Genomic polymerase chain reaction (PCR) was performed using an initial incubation at $94^{\circ} \mathrm{C}$ for $5 \mathrm{~min}$, followed by cycles of denaturation, annealing, and extension for each target gene or locus using the corresponding primer sets (Table 1 ). The reaction was terminated with a final incubation at $72^{\circ} \mathrm{C}$ for $7 \mathrm{~min}$. To confirm the target locus mutation, PCR amplicons were cloned into the pGEM-T easy vector (Promega, Madison, WI, USA) and sequenced using an ABI 3730XL DNA Analyzer (Applied Biosystems, Foster City, CA, USA).

\section{De novo assembly of RNA-sequencing data of quail transcripts}

Gene expression level was estimated through the Trinity pipeline (Version 2.2.0), with RNA-sequencing by expectation maximization (RSEM) as the transcript quantification method. We employed the Trinity pipeline for non-model organisms, mapping the reads into an assembled consensus without genomic reference to measure transcriptome levels. Trinity allows for the identification of transcript isoforms in non-model species. Prior to assembly, we employed Trimmomatic (Version 0.35) for the removal of adapter sequences. Subsequently, we performed assembly and mapping as follows. To make the reference consensus and assemble the transcriptome, we combined left and right reads in paired-end reads using 18 samples. Then, using combined left

Table 1. List of primer sets for polymerase chain reaction analysis

\begin{tabular}{lllc}
\hline Gene & Forward & Reverse & Annealing temp. $\left({ }^{\circ} \mathrm{C}\right)$ \\
\hline GAPDH & TGTCAAGGCTGAGAACGGGA & AGGCCAGGGAGCATTCTTCT & 60 \\
MyoD & ATTGGCAATGAGAGGTTCAGG & TAGAGCCTCCAATCCAGACAGA & 60 \\
Myogenin & AGCCTCAACCAGCAGGAGC & TGCGCCAGCTCAGTTTGGA & 60 \\
Desmin & CTGAAGGATGAGATGGCC & GGTCGCCTCGCTCACCAC & 60 \\
\hline
\end{tabular}

GAPDH, glyceraldehyde 3-phosphate dehydrogenase. 
and right reads, we created the assembled transcriptome with 'trinityrnaseq-2.2.0/Trinity' applying the default options. Also, we performed read mapping onto the assembled transcriptome (bowtie2) and abundance estimation (RSEM) using align_and_ estimate_abundance.pl applying the "--est_method RSEM --aln_ method bowtie --trinity_mode' options. Gene annotation was performed using TransDecoder, implemented within Trinity, and we applied several developer-recommended gene annotation tools such as blastx and blastp. Finally, using Trinotate, we extracted information on annotated genes applying the default options. To define known transcriptomic regions, we used Trinotate (http://trinotate.github.io) applying the default options.

\section{Statistical analysis}

Using the trimmed mean of M-values normalized expression as the response, the analysis of deviance model was employed to test for significance between the knockout $(\mathrm{KO})$ and control groups, as follows:

$$
\text { Expression }_{i}=\mu+\text { Group }_{\mathrm{i}}
$$

where $i=\{$ control, $\mathrm{KO}\}$ with control samples serving as the baseline. The negative-binomial assumption was considered a response variable to solve the over-dispersion problem in count data. Under the null hypothesis $\left(H_{0}\right.$ : Group $\left.=0\right)$, a likelihood ratio test was performed and the $\mathrm{p}$-values were adjusted based on false discovery rate. Here, a $1 \%$ significance level was considered significant.

\section{Reverse transcription PCR and quantitative reverse transcription-PCR}

Total RNA from undifferentiated and differentiated QM7 and MyoD KO QM7\#4 cells was isolated using Trizol Reagent (Invitrogen, USA) according to the manufacturer's instructions. RNA quality was checked by agarose gel electrophoresis and quantity was determined with a NanoDrop 2000 (Thermo Scientific, Wilmington, DE, USA). cDNA was synthesized from RNA using the Superscript III First-Strand Synthesis System (Invitrogen, USA). Each $20 \mu \mathrm{L}$ reverse transcription-PCR (RT-PCR) reaction contained $2 \mu \mathrm{L}$ cDNA, $2 \mu \mathrm{L}$ PCR buffer, $1.6 \mu \mathrm{L}$ dNTP mixture $(2.5 \mathrm{mM}), 1$ unit Taq DNA polymerase, and 10 pmol forward and reverse primer (Table 1). PCR was performed with an initial incubation at $94^{\circ} \mathrm{C}$ for $5 \mathrm{~min}$, followed by 35 cycles at $94^{\circ} \mathrm{C}$ for $30 \mathrm{~s}, 60^{\circ} \mathrm{C}$ for $30 \mathrm{~s}$, and $72^{\circ} \mathrm{C}$ for $30 \mathrm{~s}$. The reaction was terminated with a final incubation at $72^{\circ} \mathrm{C}$ for $10 \mathrm{~min}$ and the products were analyzed by agarose gel electrophoresis. For quantification of myogenic transcripts, qRT-PCR analysis was performed using the iCycler iQ Real-time PCR detection system (Bio-Rad, Hercules, CA, USA) and EvaGreen (Biotium, Fremont, CA, USA). The PCR conditions were $94^{\circ} \mathrm{C}$ for $5 \mathrm{~min}$, followed by 35 cycles at $94^{\circ} \mathrm{C}$ for $30 \mathrm{~s}, 60^{\circ} \mathrm{C}$ for $30 \mathrm{~s}$, and $72^{\circ} \mathrm{C}$ for $30 \mathrm{~s}$. Melting curve profiles were analyzed for the amplicons. Quantitative real-time PCR (qRT-PCR) data of the target genes were normalized relative to glyceraldehyde 3-phosphate dehydrogenase expression and calculated using the $2^{-\Delta \Delta C t}$ method.

\section{Western blotting}

Total protein was extracted with $1 \times$ radioimmunoprecipitation lysis buffer and separated on a $10 \%$ polyacrylamide gel followed by transfer to a nitrocellulose membrane (Bio-Rad, USA). The primary antibodies used were mouse anti- $\beta$-actin (Santa Cruz Biotechnology, Dallas, TX, USA), anti-MyoD (Santa Cruz Biotechnology, USA), anti-desmin (Novus Biologicals, Littleton, CO, USA), and anti-MF20 (Developmental Studies Hybridoma Bank, Iowa City, IA, USA). HRP-conjugated anti-mouse IgG or anti-rabbit IgG (Bio-Rad, USA) were used as secondary antibodies. The blots were treated with enhanced chemiluminescence substrate solutions and exposed in a ChemiDoc XRS System (Bio-Rad, USA) to detect chemiluminescence.

\section{RESULTS}

\section{CRISPR/Cas9-mediated MyoD knockout and establishment of single cell-derived subline}

To identify the genomic structure of the quail $M y o D$ gene, we amplified the target site with RT-PCR primers designed from chicken $M y o D$ gene sequences and performed sequencing (Figure 1A). Based on the sequence information, the target guide RNA (gRNA) site was designed and followed by a protospacer adjacent motif sequence (Figure 1A). We designed and constructed an all-in-one vector that can express both the gRNA controlled by the U6 promoter and Cas9 regulated by the CMV promoter (Figure 1B). After transfection of the Cas9-MyoD gRNA expression vector into QM7 cells, GFP-positive cells were identified by FACS and finally a single cell was isolated followed by in vitro expansion (Figure 1C). The various mutant genotypes of the mixed population were identified, and the mutant induction rate was $73.3 \%$ (11/15) (Figure 2A). The number of deleted nucleotides ranged from 1 to 28 nucleotides, and two different insertion types of 1 nucleotide were also identified (Figure 2A). We genotyped a single cell-derived subline, MyoD KO QM7\#4 with a 1-nucleotide deletion and 1-nucleotide insertion (Figure 2B). The MyoD KO QM7\#4 cell line, with single nucleotide deletion and insertion, was used in the following experiments.

\section{Characterization of QM7 MyoD KO}

In the undifferentiated state, the expression of paired box 7 (Pax7), a critical marker of undifferentiated myoblasts, in regular QM7 (rQM7) and MyoD KO QM7\#4 cells was compared by qRTPCR but no significant differences were apparent between them (Figure 3A). In addition, the morphology of the undifferentiated rQM7 and MyoD KO QM7\#4 cells was similar (Figure 3B). Under differentiation conditions, $\mathrm{rQM} 7$ cells underwent differentiation within 3 days and formed myotubes whereas MyoD KO QM7\#4 cells did not show any morphological changes even 


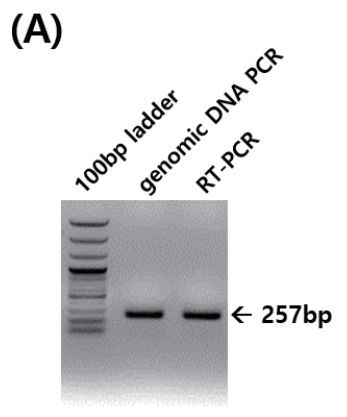

(B)

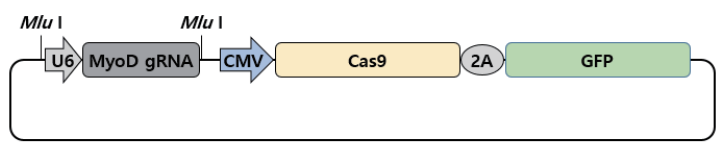

Mlu I

acgCgtTGTACAAAAAAGCAGGCTTTAAAGGAACCAATTCAGTCGACTGGATC CGGTACCAAGGTCGGGCAGGAAGAGGGCCTATTTCCCATGATTCCTTCATATT TGCATATACGATPACAAGGCTGTTAGAGAGATAATTAGAATTAATTTGACTGTA AACACAAAGATATTAGTACAAAATACGTGACGTAGAAAGTAATAATTTCTTGG U6 promoter AACACAAAGATATTAGTACAAAATACGTGACGTAGAAAGTAATAATTTCTTGG TAACTTGAAAGTATTTCGATTTCTTGGCTTTATATATCTTGTGGAAAGGACGA AACACCGCGCAAAGCCGCCACCATGAGTTTTAGAGCTAGAAATAGCAAGTTAA AATAAGGCTAGTCCGTTATCAACTTGAAAAAGTGGCACCGAGTCGGTGCTTTT guide RNA scaffold TTTCTAGACCCAGCTTTCTTGTACAAAGTTGGCATTAacgcgt

\begin{abstract}
Forward primer
target gRNA

PAM

IGCAAGGCCTGCAAGAGGAAAACCACCAATGCTGACCGCCGCAAAGCCGCCACCATGAGGGAACG GCGGCGGCTCAGCAAGGTCAATGAGGCCTTCGAGACCCTCAAGCGCTGTACCTCCACCAACCCCA CTGCTGCGCGAGCAGGAGGATGCGTACTACCCAGTGCTGGAGCACTACAGCGGGGAGTCAGA
\end{abstract}

Reverse primer
(C)

transfection with Cas9_GFP/MyoD gRNA expression vector

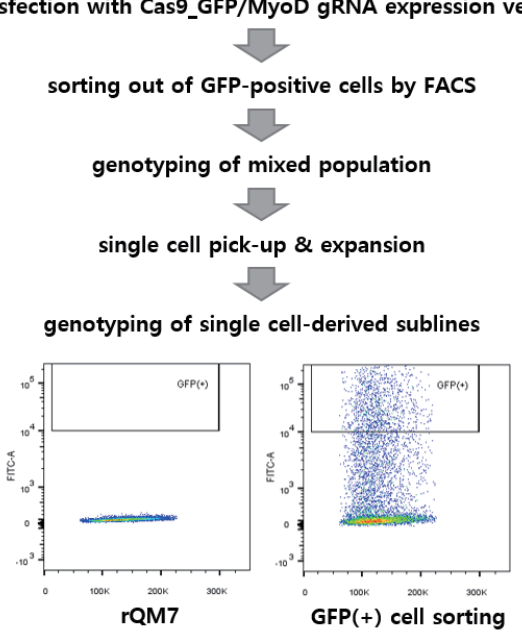

Figure 1. MyoD gene knockout in QM7 cells. (A) Genomic sequence and structure of the quail MyoD gene. (B) Expression vector of Cas9-GFP and MyoD guide RNA (gRNA). The U6 promoter controls gRNA transcription followed by a termination signal. (C) Experimental overview and fluorescence-activated cell sorting (FACS) for the development of single cellderived MyoD knockout QM7 sublines.

after 6 days of differentiation (Figure 3C). To confirm myotube formation and nuclear fusion, DAPI staining was conducted after 3 or 6 days of differentiation. Many fused nuclei were apparent in the myotubes of the differentiated rQM7 cells (Figure 4A). However, no fused nuclei or myotubes were found in MyoD KO QM7\#4 cells, even after 6 days of differentiation (Figure 4A). To confirm the expression of functional MyoD protein, Western blot analysis was performed after differentiation was induced. No functional MyoD proteins were detected in MyoD KO
QM7\#4 cells (Figure 4B).

To confirm myogenic gene induction during differentiation, RT-PCR analysis of myogenic differentiation-related genes was performed after 3 and 6 days of differentiation. MyoD transcript expression in both rQM7 and MyoD KO QM7\#4 cells was increased according to days of differentiation, although rQM7 cells showed greater expression of MyoD on day 6 compared to MyoD KO QM7\#4 cells (Figure 4C). Myogenic transcripts, including myogenin and desmin, were highly expressed in rQM7 cells but
(A)

GACCGCCGCAAAGCCGCCACCATGAGGGAACGGC
GACCGCCGCAAAGCCGCCACCA-GAGGGAACGGC
GACCGCCGCAAAGCCGCCACCA--AGGGAACGGC
GACCGCCGCAAAGCCGCCA---TGAGGGAACGGC
GACCGCCGCAAAGCCG---------GGAACGGC
GACCGCCGCAAAGCCGCC----------ACGGC
GACCG------------------------C
GACCGCCGCAAAGCCGCCACCAaTGAGGGAACGGC
GACCGCCGCAAAGCCGCCACCAtTGAGGGAACGGC

$\begin{array}{ll}\text { wild } & (4 x) \\ \text { 1nt del } & (1 x) \\ 2 n t \text { del } & (1 x) \\ \text { 3nt del } & (1 x) \\ 10 n t \text { del } & (2 x) \\ 11 n t \text { del } & (1 x) \\ 28 n t \text { in } & (1 x) \\ \text { lnt in } & (3 x) \\ \text { lnt in } & (1 x)\end{array}$

(B)

Mutation rate : $11 / 15(73.3 \%)$

Figure 2. Genotyping analysis of MyoD gene knockout QM7 cells. (A) Genotypes of MyoD-knockout mixed QM7 cells. (B) Genotypes of single cell-derived MyoD-knockout QM7 sublines (MyoD KO QM7\#4). 
(A)

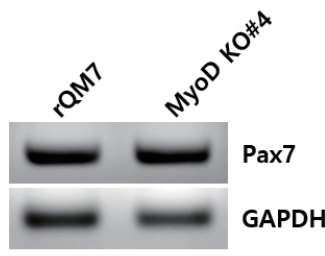

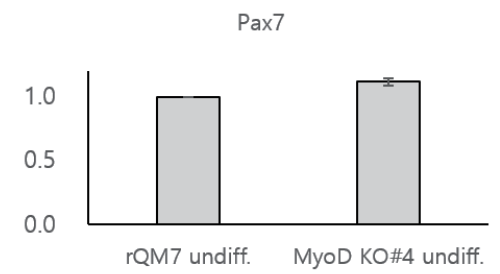

(B)

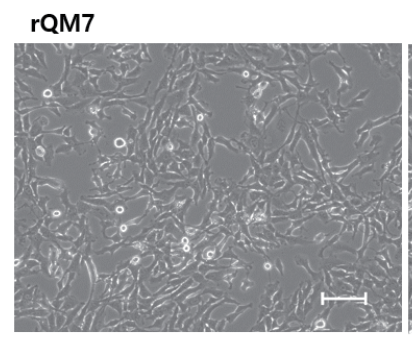

MyoD KO\#4

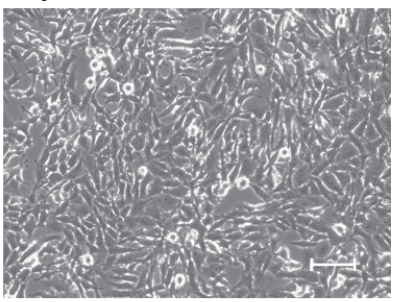

(C)

3day diff.

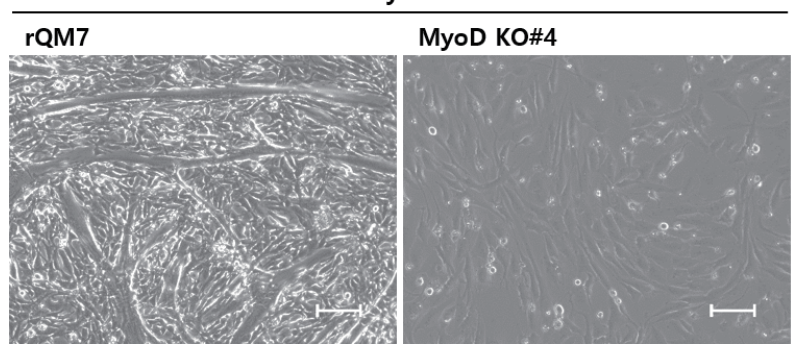

6day diff.

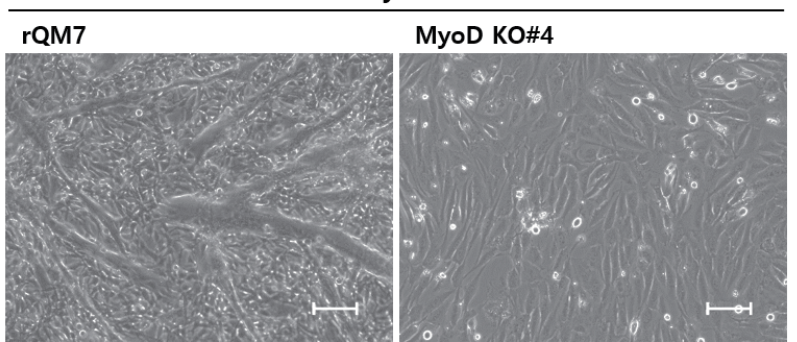

Figure 3. Characterization of MyoD-knockout QM7 cells. (A) Paired box 7 (Pax7) expression in regular QM7 (rQM7) and MyoD-knockout QM7 (MyoD KO QM7\#4) cells. There were no significant differences between rQM7 and MyoD KO QM7\#4 cells. (B) Morphology of undifferentiated rQM7 and MyoD KO QM7\#4 cells. (C) Morphological changes 3 or 6 days after differentiation. rQM7 cells transformed into myotubes during differentiation. No morphological changes were evident in MyoD KO QM7\#4 cells after 6 days of differentiation. All images were observed under the inverted microscope, scale bars $=200 \mu \mathrm{m}$.

(A)
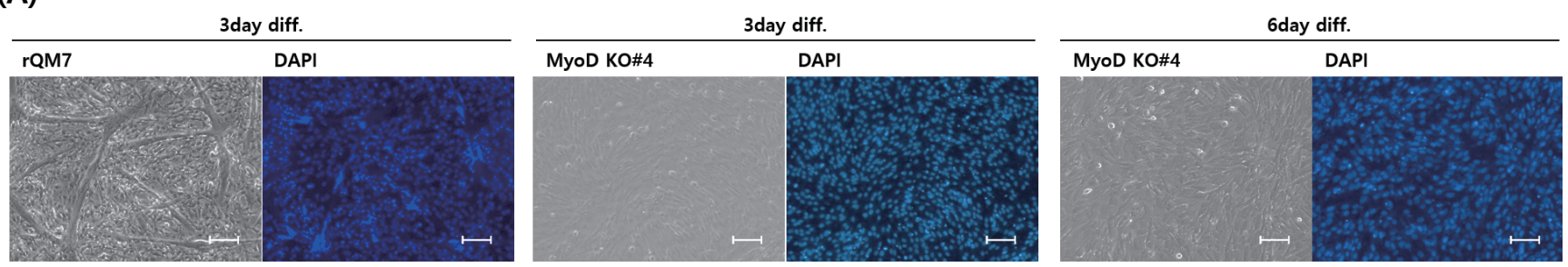

(B)

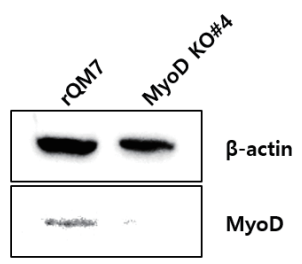

(D)

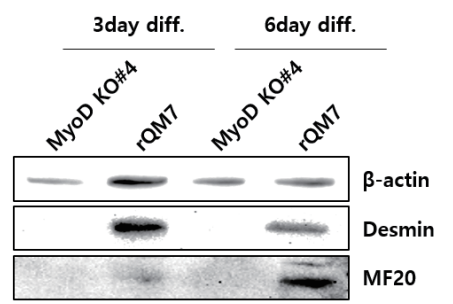

(C)
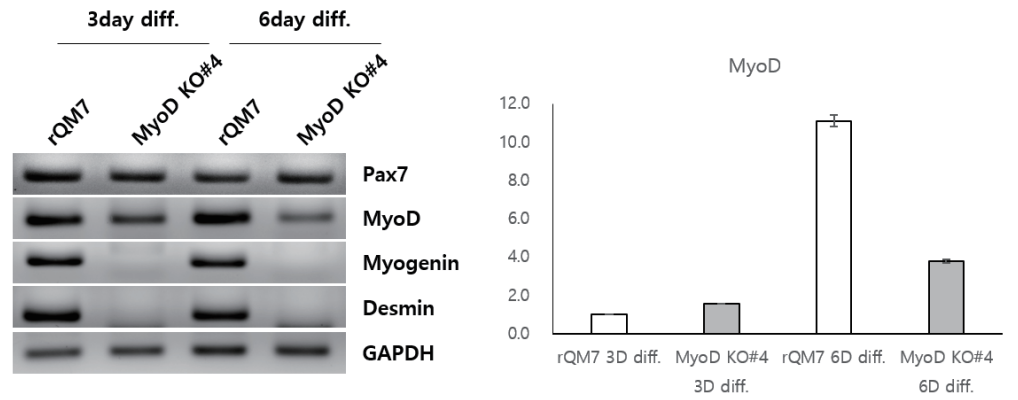

Figure 4. (A) Nuclear fusion in regular QM7 (rQM7) and MyoD-knockout QM7 (MyoD KO QM7\#4) cells during myotube differentiation. No fusion was evident in MyoD KO QM7\#4 cells even after 6 days of differentiation. (B) Western blotting detected MyoD protein in rQM7 and MyoD KO QM7\#4 cells after 3 days of differentiation. (C) RT-PCR analysis of myogenic differentiation-related genes and quantitative RT-PCR of the MyoD gene in RQM7 and MyoD KO QM7\#4 cells during differentiation. (D) Detection of myogenic differentiation-related genes in rQM7 and MyOD KO QM7\#4 cells during differentiation. RT-PCR, reverse transcription-polymerase chain reaction. The images were observed under the inverted fluorescent microscope after staining with 4',6-Diamidine-2'-phenylindole dihydrochloride (DAPI), scale bars = $200 \mu \mathrm{m}$. 
were not detected in MyoD KO QM7\#4 cells even after 6 days of differentiation (Figure 4C). Western blot analysis revealed myogenic terminal proteins such as desmin and myosin heavy chain (MF20) in the differentiated rQM7 cells, but not in the differentiated MyoD KO QM7\#4 cells (Figure 4D).

\section{Global gene expression analysis by RNA sequencing in QM7 MyoD KO cells}

Because there is no public genome information on quail, de novo assembly was performed following RNA sequencing of the differentiated rQM7 and MyoD KO QM7\#4 sublines. Figure 4 shows string analysis of differentially expressed genes (DEGs) of rQM7 and MyoD KO QM7\#4 cells. DEGs that were significantly lower in MyoD KO QM7\#4 cells during differentiation are shown in Figure 5. The majority of myogenic genes were dramatically downregulated and the regulatory pathway was repressed in $\mathrm{MyoD}$ KO QM7\#4 cells during differentiation. Critical myogenic genes involved in myotube differentiation including Pax7, myogenin (MyoG), myosin heavy chain 1 (MYH1), hairy/enhancer-of-split related with YRPW motif protein 1 (Hey1), DNA-binding protein inhibitor ID1 (ID1), and activin A receptor type I (ACVR1) were precipitously downregulated in MyoD KO QM7\#4 cells (Table 2).

\section{DISCUSSION}

The CRISPR/Cas9 system, an incredibly efficient and reliable tool for targeted genomic modification of living cells, has generated considerable excitement for industrial applications as well as for basic scientific research $[13,14]$. This technical platform has revolutionized bioscience and biotechnology because of its simplicity and highly efficient editing of the genome [21-23]. Although knockout chickens have been produced by the con-

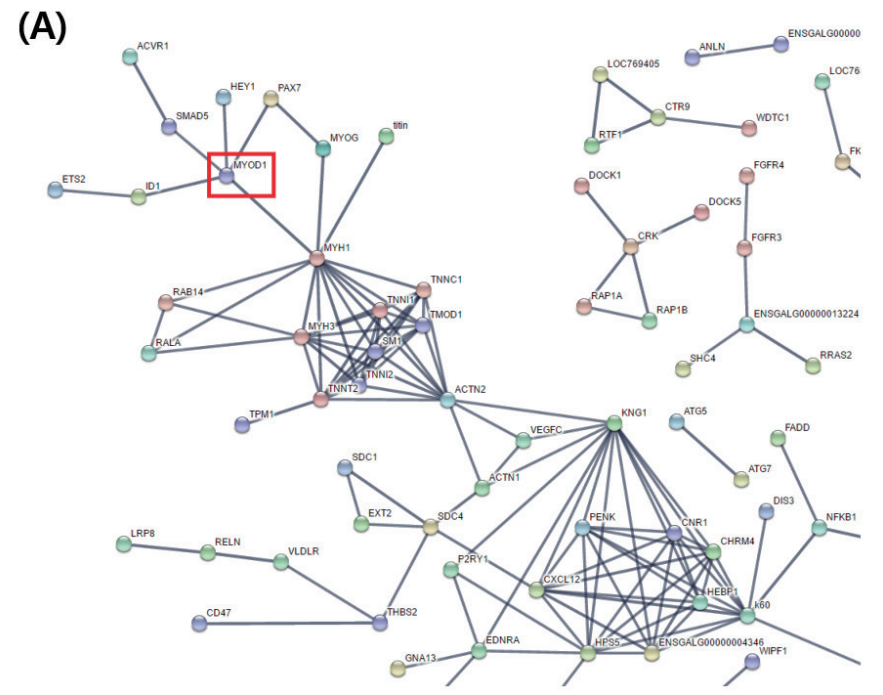

Table 2. List of top 30 genes down-regulated in the differentiated MyoD KO QM7\#4 compared to those of the differentiated rQM7

\begin{tabular}{llc}
\hline Gene & Description & $\begin{array}{c}\text { Fold change } \\
\text { (Log ratio) }\end{array}$ \\
\hline TNNT2 & Troponin T, cardiac muscle isoforms & -17.4203 \\
MLRB & Myosin regulatory light chain 2B, cardiac muscle isoform & -17.3022 \\
MYOG & Myogenin & -15.9997 \\
MYBPH & Myosin-binding protein H & -15.6882 \\
CASQ2 & Calsequestrin-2 & -15.150 \\
ACHG & Acetylcholine receptor subunit gamma & -14.8215 \\
MLE1 & Myosin light chain 1, skeletal muscle isoform & -14.6863 \\
DESM & Desmin & -14.2803 \\
CAV3 & Caveolin-3 & -14.2394 \\
EF1A2 & Elongation factor 1-alpha 2 & -13.9406 \\
TNNI1 & Troponin I, slow skeletal muscle & -13.8945 \\
DUS26 & Dual specificity protein phosphatase 26 & -13.8884 \\
MLEC & Myosin light chain 1, cardiac muscle & -13.5580 \\
KCRM & Creatine kinase M-type & -13.3336 \\
DDR1 & Epithelial discoidin domain-containing receptor 1 & -13.2175 \\
MYOZ2 & Myozenin-2 & -12.8902 \\
TNNT2 & Troponin T, cardiac muscle & -12.8211 \\
MLE3 & Myosin light chain 3, skeletal muscle isoform & -12.7211 \\
TNNI2 & Troponin I, fast skeletal muscle & -12.6837 \\
TBA3 & Tubulin alpha-3 chain & -12.5555 \\
POPD1 & Blood vessel epicardial substance & -12.5325 \\
LOLA1 & Longitudinals lacking protein, isoform G & -12.4171 \\
TBA3 & Tubulin alpha-3 chain & -12.3884 \\
TBA1C & Tubulin alpha-1C chain & -12.1987 \\
HSPB2 & Heat shock protein beta-2 & -12.1131 \\
TM182 & Transmembrane protein 182 & -11.8558 \\
SH3BG & SH3 domain-binding glutamic acid-rich protein & -11.8005 \\
MNME & tRNA modification GTPase & -11.7358 \\
MYH6 & Myosin-6 & -11.5455 \\
MALQ & 4-alpha-glucanotransferase & -11.5129 \\
\hline & & \\
& &
\end{tabular}

ventional homologous recombination gene targeting and transcription activator-like effector nuclease technique [7], the

(B)

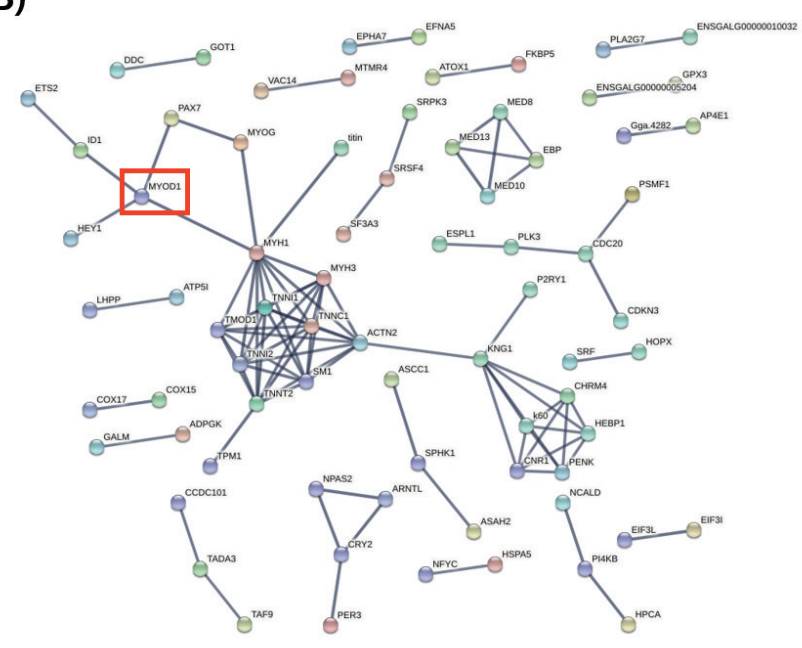

Figure 5. String analysis of differentially expressed genes (DEGs) processed by RNA-sequencing data. (A) String analysis of total DEGs of rQM7 and MyoD KO QM7\#4 cells during differentiation. (B) String analysis of DEGs significantly decreased in MyoD KO QM7\#4 cells during differentiation. The red box indicates the MyoD transcript. 
versatile CRISPR/Cas9 system is more efficient, particularly for large-scale and high-throughput, precise genetic changes in avian species, and is not time-consuming or complex. In this study, we knocked out a myogenic gene and investigated a regulatory pathway that could be applied to improve traits such as growth and meat production in the poultry industry.

For differentiation experiments, we used the MyoD KO QM7\#4 subline with two single nucleotide changes, a nucleotide insertion and deletion, in the $M y o D$ gene (Figure 2B) to examine the single nucleotide changes frequently detected during the breedingselection process. The expression of Pax7, a critical marker of undifferentiated myoblasts, was similar between rQM7 and MyoD KO QM7\#4 cells (Figure 3A), indicating that deletion of the $M y o D$ gene did not affect myogenic integrity in the undifferentiated state. However, MyoD KO QM7\#4 cells did not transform into myotubes when differentiation was induced (Figures 3,4). Through RNA sequencing analysis, we determined that several myogenic genes, including MyoG and MYH1, were repressed in MyoD KO QM7\#4 cells compared to rQM7 cells (Figure 5B, Table 2). Heyl is a nuclear protein that belongs to the hairy and enhancer of split-related family of helix-loop-helix (HLH) type of transcriptional repressors [24]. This gene family is required for cardiovascular development in mouse embryos [24]. ID1 is also an HLH protein that can form heterodimers with other HLH family members [25] and may play a role in cell growth, senescence, and differentiation [26,27]. The ACVR1 gene, also downregulated in differentiated MyoD KO QM7\#4 cells, plays an important role in the bone morphogenic protein pathway, which is responsible for the development and repair of the skeletal system [28]. ACVR1 is a differentiation factor that belongs to the transforming growth factor-beta superfamily [28]. Thus, in this study, we examined the changes in the gene regulatory network caused by knockout of the $M y o D$ gene during myoblast differentiation. Although MyoD-knockout mice lack functional MyoD, they are still viable and fertile $[19,20]$. They also do not exhibit any morphological or physiological abnormalities in skeletal muscle $[19,20]$. The expression levels of skeletal musclespecific genes in MyoD-knockout mice are similar to those of wild-type mice $[19,20]$. However, in our study, myotube differentiation was repressed in MyoD-knockout quail myoblast cells, and these cells did not express any myogenic transcripts or proteins (Figure 4C, 4D). Despite these differences, the morphologies of the MyoD-knockout cells were similar to wild-type myoblasts (Figure 3B). This may be explained by differences in the myogenic regulatory network between mammal and avian species. Future studies will need to generate MyoD-knockout poultry to further investigate the regulatory mechanisms of skeletal muscle development in avian species.

$\mathrm{MyoD}$ is a prerequisite for myogenic initiation and it stably commits the muscle cell to differentiation. In this study, we clearly present the biofunctionality of MyoD using CRISPR/Cas9mediated knockout avian myoblasts. Our results also demonstrate that use of the CRISPR/Cas9 technical platform facilitates functional analyses of specific genes in avian cell lines. The development of specific, genome-tailored knockout cell lines will help advance bioscience, in particular the production of avian models. In addition, the generation of genome-edited poultry mediated by the CRISPR/Cas9 system will be widely utilized in agriculture and industry.

\section{CONFLICT OF INTEREST}

We certify that there is no conflict of interest with any financial organization regarding the material discussed in the manuscript.

\section{ACKNOWLEDGMENTS}

This work was carried out with the support of "Cooperative Research Program for Agriculture Science \& Technology Development (Project No. PJ01111401)" Rural Development Administration, Republic of Korea. This work was also supported by Korea Institute of Planning and Evaluation for Technology in Food, Agriculture, Forestry and Fisheries (IPET) through Agri-Bio Industry Technology Development Program, funded by Ministry of Agriculture, Food and Rural Affairs (MAFRA) (316005-5).

\section{REFERENCES}

1. Hillier LW, Miller W, Birney E et al. Sequence and comparative analysis of the chicken genome provide unique perspectives on vertebrate evolution. Nature 2004;432:695-716.

2. Rubin CJ, Zody MC, Eriksson J, et al. Whole-genome resequencing reveals loci under selection during chicken domestication. Nature 2010;464:587-91.

3. Al-Musawi SL, Lock F, Simbi BH, Bayol SAM, Stickland NC. Muscle specific differences in the regulation of myogenic differentiation in chickens genetically selected for divergent growth rates. Differentiation 2011;82:127-35.

4. Shin S, Song Y, Ahn J, et al. A novel mechanism of myostatin regulation by its alternative splicing variant during myogenesis in avian species. Am J Physiol Cell Physiol 2015;309:650-9.

5. Park TS, Han JY. Genetic modification of chicken germ cells. Ann NY Acad Sci 2012;1271:104-9.

6. Park TS, Han JY. piggyBac transposition into primordial germ cells is an efficient tool for transgenesis in chickens. Proc Natl Acad Sci USA 2012;109:9337-41.

7. Park TS, Lee HJ, Kim KH, Kim JS, Han JY. Targeted gene knockout in chickens mediated by TALENs. Proc Natl Acad Sci USA 2014;111: 12716-21.

8. Salter DW, Smith EJ, Hughes SH, et al. Gene insertion into the chicken germ line by retroviruses. Poult Sci 1986;65:1445-8.

9. Love J, Gribbin C, Mather C, Sang H. Transgenic birds by DNA microinjection. Biotechnology 1994;12:60-3.

10. Macdonald J, Taylor L, Sherman A, et al. Efficient genetic modifi- 
cation and germ-line transmission of primordial germ cells using piggyBac and Tol2 transposons. Proc Natl Acad Sci USA 2012;109: E1466-E72.

11. Schusser B, Collarini EJ, Yi H, et al. Immunoglobulin knockout chickens via efficient homologous recombination in primordial germ cells. Proc Natl Acad Sci USA 2013;110:20170-5.

12. Dimitrov L, Pedersen D, Ching KH, et al. Germline gene editing in chickens by efficient CRISPR-mediated homologous recombination in primordial germ cells. PLoS ONE 2016;11:e0154303.

13. Cong L, Ran FA, Cox D, et al. Multiplex genome engineering using CRISPR/Cas systems. Science 2013;339:819-23.

14. Mali P, Yang L, Esvelt KM, et al. RNA-guided human genome engineering via Cas9. Science 2013;339:823-6.

15. Davis RL, Weintraub H, Lassar AB. Expression of a single transfected cDNA converts fibroblasts to myoblasts. Cell 1987;51:987-1000.

16. Rudnicki MA, Schnegelsberg PNJ, Stead RH, et al. MyoD or Myf-5 is required for the formation of skeletal muscle. Cell 1993;75:1351-9.

17. Berkes CA, Tapscott SJ. MyoD and the transcriptional control of myogenesis. Semin Cell Dev Biol 2005;16:585-95.

18. Buckingham M. Myogenic progenitor cells and skeletal myogenesis in vertebrates. Curr Opin Genet Dev 2006;16:525-32.

19. Rudnicki MA, Braun T, Hinuma S, Jaenisch R. Inactivation of $M y o D$ in mice leads to up-regulation of the myogenic HLH gene $M y f-5$ and results in apparently normal muscle development. Cell 1992;71: 383-90.
20. Arnold HH, Braun T. Targeted inactivation of myogenic factor genes reveals their role during mouse myogenesis: a review. Int J Dev Biol 1996;40:345-53.

21. Wang H, Yang H, Shivalila CS, et al. One-step generation of mice carrying mutations in multiple genes by CRISPR/Cas-mediated genome engineering. Cell 2013;153:910-8.

22. Jao LE, Wente SR, Chen W. Efficient multiplex biallelic zebrafish genome editing using a CRISPR nuclease system. Proc Natl Acad Sci USA 2013;110:13904-9.

23. Hai T, Teng F, Guo R, Li W, Zhou Q. One-step generation of knockout pigs by zygote injection of CRISPR/Cas system. Cell Res 2014;24: $372-5$.

24. Weber D, Wiese C, Gessler M. Hey bHLH transcription factors. Curr Top Dev Biol 2014;110:285-315.

25. Benezra R, Davis RL, Lockshon D, Turner DL, Weintraub H. The protein Id: a negative regulator of helix-loop-helix DNA binding proteins. Cell 1990;61:49-59.

26. Ruzinova MB, Benezra R. Id proteins in development, cell cycle and cancer. Trends Cell Biol 2003;13:410-8.

27. Perk J, Iavarone A, Benezra R. The Id family of helix-loop-helix proteins in cancer. Nat Rev Cancer 2005;5:603-14.

28. Kaplan FS, Xu M, Seemann P, et al. Classic and atypical fibrodysplasia ossificans progressiva (FOP) phenotypes are caused by mutations in the bone morphogenetic protein (BMP) type I receptor ACVR1. Hum Mutat 2009;30:379-90. 